Julián Fernández Mejía* Jorge Mario Unibe

Universidad del Valle, Cali, Colombia

Recibido: 17 de junio de 2015

Concepto de evaluación: 18 de noviembre de 2015

Aprobado: 9 de diciembre de 2015

Artículo de investigación (C) 2016 Universidad Católica de Colombia. Facultad de Ciencias Económicas y Administrativas. Todos los derechos reservados

* Estudiante de la Maestría en Economía Aplicada y economista por la Universidad del Valle. Integrante del Grupo de Macroeconomía Aplicada y Economía Financiera y del Grupo de Logística y Producción de esa misma universidad. Joven investigador Colciencias. Dirección de correspondencia: calle 13 \# 100-00,

Ciudad Universitaria Meléndez, Edificio 387, Departamento de Economía, Cali, Colombia. Correo electrónico: julian.fernandez.mejia@ correounivalle.edu.co

** Magíster del Instituto Europeo y la

Universidad de los Andes. Profesor del Departamento de Economía de la Universidad del Valle, Cali, Colombia. Integrante del Grupo de Macroeconomía Aplicada y Economía Financiera de esa misma universidad. Correo electrónico: jorge.uribe@ correounivalle.edu.co

\section{Análisis de procesos explosivos en el precio de los activos financieros: evidencia alrededor del mundo*}

\section{RESUMEN}

En este artículo se analizan diferentes índices accionarios de mercados alrededor del mundo, en el periodo 1995-2013, con el fin de poner a prueba la existencia y fechar la aparición de procesos explosivos en sus mercados de acciones. Se hace uso de una prueba de signo, para construir diferentes índices de burbujas en los mercados financieros representativos de cada región, y se construye además un índice de las principales regiones financieras a partir de modelos dinámicos por factores. Estos índices permiten caracterizar las regiones en términos de riesgo y, asimismo, de ocurrencia de burbujas financieras. Se encuentra evidencia que señala cierto grado de sincronización entre los episodios de burbujas financieras en los mercados analizados y, en general, en todo el mundo.

Palabras claves: burbujas, prueba de signo, factores, índices, crisis.

JEL : G01, G14, C22, C15, C18, C58

\section{Analysis of financial asset price explosions: Evidence from around the world}

\section{ABSTRACT}

This article analyzes different international share price indices for the period 1995-2013, in order to test for the existence and date of appearance of asset price explosions in the world's stock markets. A sign test is employed to construct different indices of bubbles in representative financial markets for each region, using dynamic factor models. These indices permit a

\footnotetext{
* Los autores agradecen los valiosos comentarios y aportes realizados por los dos califica-
} dores y los editores en la elaboración de la versión final del artículo. 
characterization to be made of each region in terms of risk and, also, of the occurrence of financial bubbles. Evidence is found that indicates a certain degree of synchronization between episodes of financial bubbles in the markets analyzed and, generally, at international level.

Keywords: bubbles, sign test, factors, indices, crises.

\section{Análise de processos explosivos no preço dos ativos financeiros: evidência ao redor do mundo}

\section{RESUMO}

Neste artigo, analisam-se diferentes índices acionários de mercados ao redor do mundo, no período 1995-2013, com o objetivo de avaliar a existência e datar a aparição de processos explosivos em seus mercados de ações. Utilizase um teste de signo para construir diferentes índices de bolhas nos mercados financeiros representativos de cada região e constrói-se, além disso, um índice das principais regiões financeiras a partir de modelos dinâmicos por fatores. Esses índices permitem caracterizar as regiões em termos de risco e, desse modo, de ocorrência de bolhas financeiras. Encontra-se evidência que aponta certo grau de sincronização entre os episódios de bolhas financeiras nos mercados analisados e, em geral, em todo o mundo.

Palavras-chave: bolhas, teste de signo, fatores, índices, crise. 


\section{INTRODUCCIÓN}

Las burbujas en los precios de los activos financieros, así como el contagio financiero internacional, son temas de extenso escrutinio en la literatura económica contemporánea. Ambos se encuentran de forma recurrente en el debate académico y en el centro de las decisiones de política económica de los gobiernos. También son de especial interés para los formuladores de la política monetaria, puesto que su aparición está relacionada con episodios de alta volatilidad y de crisis sistémicas en los mercados financieros. Por tanto, avanzar en la comprensión de estos fenómenos promete contribuir con el diseño de herramientas de política y de administración de portafolios, que ayuden a aminorar los efectos adversos que las crisis financieras traen consigo, en términos de riqueza y bienestar para los agentes de mercado, o al menos iluminar nuestro entendimiento sobre qué tan deseables o factibles son tales herramientas.

En ambos frentes se han hecho importantes avances en épocas recientes. En una contribución actual, Brunnermeier y Oehmke (2013) resumen distintos vínculos que existen entre las burbujas, el riesgo sistémico y las crisis financieras. Tal y como los autores señalan, a pesar del alto componente idiosincrático de cada boom y de las sendas de crisis en las economías de mercado, en la anatomía típica de una crisis financiera, un periodo de expansión pronunciada en los precios de los activos, impulsado inicialmente por algún cambio en los fundamentales de la economía o por una innovación financiera, engendra la aparición de una burbuja. A esta expansión la sigue una caída abrupta en tales precios, en la que cobran relevancia mecanismos de amplificación de los mercados que se encuentran latentes en épocas de regularidad financiera.

Esta dinámica de expansión y contracción contribuye de forma importante a la generación de ciclos financieros, que al sincronizarse con los ciclos reales de la economía, llevan a contracciones severas de la actividad económica real y, por tanto, a pérdidas de bienestar significativas para la sociedad. Por lo anterior, no resulta extraño que las burbujas financieras siempre sean un tema que genera inmensa atracción en la literatura económicofinanciera. Su estudio se enlaza profundamente con las raíces de gran parte de las teorías modernas del pensamiento económico y de los desarrollos empíricos logrados en diferentes corrientes de la economía como ciencia.

El primer paso que se debe garantizar en el análisis de la sobrevaloración de activos es el de lograr la identificación temporal de las burbujas y su conceptualización, es decir, definir el cuándo y el porqué de su existencia. El estudio del porqué se fundamenta en los análisis teóricos sobre la posibilidad misma de tales fenómenos. En esta corriente de la literatura ha sido particularmente difícil hacer coherente un esquema de modelación de equilibrio general, con el supuesto de expectativas racionales tradicional, a la Lucas (Tirole, 1982). De ahí que a las burbujas se les obligó a habitar en los terrenos del equilibrio parcial (Allen y Gale, 2000) o de generaciones traslapadas (Tirole, 1985), donde han estado presentes desde Samuelson (1958) y Shell (1971) resolviendo ineficiencias dinámicas en la economía, encarnadas en el dinero fiduciario.

El otro camino que se les obligó a transitar a las burbujas teóricas fue al margen de la hipótesis de las expectativas racionales. En esta segunda vertiente de la literatura, que cada vez cobra más relevancia, gran parte de las teorías desarrolladas parten de la existencia de fenómenos comportamentales, según los cuales las burbujas están asociadas con conductas que no son racionales, en el sentido más estricto del término. Se puede decir, entonces, que están asociadas con "espíritus animales", como los descritos por Keynes (1936) y posteriormente estudiados por Akerlof y Shiller (2009), Shell (1971) y Shiller (2005).

En esta literatura, tales espíritus hacen referencia a los aspectos emocionales que afectan la confianza de los agentes, que se pueden ver avocados a tomar decisiones riesgosas (o más riesgosas de lo que sería óptimo en situaciones de regularidad de mercados) frente a una expectativa de retorno futuro (Farmer, 2012). En la mayoría de los casos, las expectativas futuras de los precios de 
los activos no están fundamentadas en indicadores de desempeño, conocidos como fundamentales, sino que revisten un carácter especulativo, que para muchos observadores externos puede parecer irracional.

El presente artículo se centra en definir el cuándo de las burbujas financieras globales, es decir, en la medición empírica de los momentos de aparición y de colapso de distintas burbujas financieras, en diferentes mercados de acciones, alrededor del mundo, en décadas recientes. El estudio simultáneo del fenómeno en diferentes mercados permitirá dilucidar mecanismos y factores globales latentes que pudieran estar proveyendo el combustible de las burbujas futuras. También permitirá sentar las bases para un primer análisis de oportunidades reales de diversificación que existen para los inversionistas globales, que deseen evitar el riesgo de colapso implícito en estos fenómenos, cuando tal posibilidad existe.

La estrategia de modelación empírica se basa en un análisis de series de tiempo, de las características de explosividad que revisten los índices temporales de los mercados de acciones. Para ello, el artículo hace uso de una metodología explorada en varios documentos de la literatura nacional (Uribe, 2013; Uribe y Fernández, 2014a; Uribe y Ulloa, 2014), que se alinea bastante con la lógica de recientes avances en la econometría de series de tiempo en el ámbito mundial (Anderson, Brooks y Katsaris, 2010; Anderson y Brooks, 2014; Homm y Breitung, 2012; Phillips, Shi y Wu, 2014; Phillips, Wu y Yu, 2011a, 2011b; Shi, 2007; Sornette, Woodard y Zhou, 2009a; Yuhn, Kim y Nam, 2015; Zhou y Sornette, 2005, 2003).

En todos los casos, la idea básica es que las burbujas pueden ser detectadas estadísticamente, porque, o bien dejan huellas rastreables que pueden ser modeladas a través de leyes de potencia tradicionales (o quiebres estructurales en los coeficientes que las rigen), o bien, están asociadas con situaciones de crecimiento acelerado de los precios, que llevan a transitar desde el mundo de las raíces unitarias hacia el mundo de la explosividad en el tiempo. Identificar en estos casos la burbuja consiste en estimar en qué momento el proceso de precios deja de ser una raíz unitaria, y se vuelve un proceso explosivo.

Este documento propone, entonces, varios avances en la literatura. Primero, expande la muestra de estudio de Uribe y Fernández (2014a), autores que analizan el caso de algunos mercados en Latinoamérica y encuentran evidencia de sincronización en las burbujas de los mismos. No obstante, su análisis deja por fuera la dinámica experimentada por las economías desarrolladas en su conjunto, y por muchas otras economías emergentes, que brinda mayores luces sobre lo acontecido en las recientes crisis financieras globales, en términos de sobrevaloración. Debido a esta muestra de estudio, considerablemente más amplia, nuestras estimaciones nos permiten identificar con mayor precisión el mercado de acciones global en el que el fenómeno de sobrevaloración se generó en primer lugar; es decir, el centro de la inestabilidad global recientemente experimentada en el mundo, aun con secuelas reales en marcha.

Un segundo aporte de este artículo es la construcción de índices por regiones de sobrevaloración de activos. Estos índices ayudan a sintetizar la información contenida en las estimaciones individuales y pueden ser de gran utilidad para inversionistas que determinen su posición de portafolio con base en consideraciones geográficas, de regiones, en lugar de hacerlo por países. Los índices también pueden ser interpretados como un indicador de riesgo financiero. Además, cada índice regional permite discernir el grado de sincronización que posee cada mercado en relación con el ámbito mundial. En la medida en que se encuentran burbujas en los mismos periodos, se puede determinar el grado de relación que existe entre un mercado y otro, además de las oportunidades de diversificación eficiente del portafolio.

Dentro de este trabajo se encuentra evidencia de sincronización, en diferentes etapas, de los procesos de burbujas en todo el mundo, especialmente durante los periodos de las burbujas punto-com y sub-prime, que permiten determinar la existencia de crisis financieras generalizadas 
y cierta homogeneidad en sus efectos en las diferentes regiones. Se encuentra además que dentro de las diferentes crisis financieras, originadas principalmente en el mercado estadounidense, la burbuja inmobiliaria que generó la crisis de 2007 ha sido la que trajo consigo los mayores efectos sobre los mercados internacionales. Esto supone la necesidad de controlar los canales de transmisión de las crisis, desde esta economía hacia las demás, y de adoptar políticas conducentes a atenuar los efectos adversos sobre el mercado financiero global.

El artículo está constituido por cuatro secciones. En la primera se presenta un breve estado del arte de las metodologías más usadas para el análisis de las burbujas financieras. En la segunda se consigna la metodología econométrica empleada; allí se explica el proceso de estimación a través de la prueba de signo, así como el proceso de construcción del índice de burbujas, mediante índices de difusión. En la tercera se presentan los datos y los resultados obtenidos. Por último, se presentan las conclusiones.

\section{LITERATURA EN BURBUJAS FINANCIERAS}

Desde hace varios años, la medición de burbujas financieras en los precios de los activos ha sido un tema de gran importancia en la literatura económica mundial. La medición de estos fenómenos ha llevado al uso de múltiples metodologías para determinar la existencia de fenómenos especulativos en las series financieras. Se pueden categorizar las diferentes metodologías, siguiendo a Gürkaynak (2008), en cuatro grupos: a) pruebas de acotamiento en la varianza, b) etapas de West, c) integración y cointegración de burbujas intrínsecas y d) burbujas como variables no observables. Además de estas categorías, existen dos adicionales: por una lado, las que señalan las burbujas como un proceso de cambio de régimen en los precios de los activos y, por otro, las que modelan las burbujas a partir de modelos de física estadística aplicados a la economía.

La primera categoría, denominada prueba de acotamiento de varianza, fue propuesta originalmente por Shiller (1981) y LeRoy y Porter (1981).
La metodología consiste en el análisis de la diferencia entre las varianza de los precios futuros (medidos como el valor presente de los dividendos futuros) y el precio actual (medido como el valor presente de los dividendos esperados). De acuerdo con esta relación, bajo expectativas racionales, la varianza futura debería ser mayor o igual a la presente, es decir, debería estar acotada inferiormente por la varianza actual. Cualquier desviación pude ser interpretada como un exceso de varianza y, por tanto, podría estar asociada con fenómenos especulativos y la presencia de burbujas en el mercado. Siguiendo esta idea, otros artículos como los de West (1988) y Campbell y Shiller (1988a, 1988b) ejecutan la misma prueba, aunque realiza algunas mejoras al modelo original.

El segundo tipo de pruebas corresponde a las medidas en dos etapas de West (1987). Esta prueba, como su nombre lo dice, analiza a partir de dos fases la existencia de burbujas en el mercado. La primera etapa realiza una prueba de especificación sobre el modelo, y la segunda etapa corresponde a una prueba que permite descartar la existencia de burbujas.

El tercer tipo de pruebas, conocidas como las pruebas de integración y cointegración, buscan determinar si existe un comportamiento explosivo en las series. De acuerdo con este comportamiento explosivo, se podría determinar la burbuja en el mercado. Diba y Grossman (1987, 1988a, 1988b) plantean que, dada la condición de no arbitraje en los mercados eficientes, los precios deberían tener una relación de cointegración con los dividendos. De no existir tal relación de cointegración, se encontraría evidencia de la existencia de burbujas financieras en el mercado.

A partir de este modelo, surge una de las principales críticas, que sería el fundamento de las medidas posteriores de burbujas: la crítica de Evans (1991). El autor menciona que, en toda la serie, las pruebas de cointegración y de integración establecen una relación de largo plazo; sin embargo, la existencia de burbujas que presentan comportamientos explosivos en plazos menores puede inducir, con una alta frecuencia, al rechazo 
de la hipótesis de existencia de burbujas, aun cuando esta sea verdadera.

Esta crítica llevó a los desarrollos de pruebas como las de Phillips, Shi y Yu (2014), Phillips, Wu y Yu (2011) y Phillips y Yu (2011), quienes, a partir de la prueba Dickey-Fuller Aumentada (Dickey y Fuller, 1979) con ventanas móviles, construyen un índice de burbujas para el mercado estadounidense. De la misma manera, Uribe y Fernández (2014) y Uribe (2013) desarrollan un test de burbujas a partir del test de signo de So y Shin (2001) para fechar burbujas financieras, en el que se basa este estudio.

La quinta categoría corresponde a las pruebas para detectar burbujas intrínsecas en los precios de los activos, desarrolladas por Froot y Obstfeld (1991). Estas burbujas están relacionadas con los fundamentales, es decir, no son independientes del comportamiento de los dividendos mismos. Esta prueba se diferencia de las demás por cuanto la aparición de la burbuja está atada a la diferencia entre los dividendos y el proceso de la burbuja, teniendo en cuenta que se trata de series que no son independientes estadísticamente. Los autores diseñan una prueba que se encarga de detectar la existencia de burbujas y determinan relaciones no lineales entre los precios y los dividendos. El supuesto principal es que los precios se mueven bajo una relación lineal con los dividendos y, por tanto, cualquier divergencia estará dada por el comportamiento explosivo proveniente del componente de burbuja.

La última categoría presentada por Gürkaynak (2008) corresponde a las pruebas de burbujas como variables no observables propuestas por Wu (1997). Para esto, teniendo en cuenta el modelo de valoración de los dividendos, como un comportamiento autorregresivo, el autor modela la variable no observable (burbuja) mediante la representación estado-espacio que permite esta metodología. No obstante, esta medida ha sido criticada, pues aunque logra identificar el componente no observado, este también puede representar una mala especificación del modelo.

Los modelos basados en la medida de cambio de régimen (Hamilton, 1989) permiten modelar los cambios en los procesos generadores de datos de los precios de los activos. Así, estas pruebas permiten superar la crítica de Evans (1991) e identificar el proceso de burbuja en los datos (Hall, Psaradakis y Sola, 1999; Hall y Sola, 1993; Van Norden y Vigfusson, 1998; Van Norden, 1996). Pero esta modelación ha sido criticada, ya que empíricamente es difícil diferenciar entre un cambio de régimen en los dividendos de uno generado por las burbujas en los precios.

Finalmente, métodos de aparición reciente son las pruebas de burbujas basadas en aplicaciones de los modelos de física estadística a los datos financieros, propuestos por Corsi y Sornette (2014), Leiss, Nax y Sornette (2015), Malevergn y Sornette (2006), Sornette, Woodard y Zhou (2009b), Sornette (2009), entre otros. Para llevar a cabo la modelación de las series de tiempo, estos modelos utilizan las leyes de potencia tradicionales y cambios en los parámetros que las describen, con lo que logran cierto éxito no solo en la modelación de las burbujas, sino también en la predicción de su colapso.

\section{METODOLOGÍA}

\section{Prueba de signo}

En este trabajo se hace uso de la prueba de signo propuesta por So y Shin (2001) para detectar caminatas aleatorias versus procesos alternativos generales y no estacionarios. Esta prueba, a diferencia de otras utilizadas para el mismo fin, es consistente ante una amplia gama de procesos no lineales, estacionarios o explosivos, y además posee mejores propiedades en términos de poder asintótico local en comparación con alternativas tradicionales. Se debe mencionar que también es invariante ante transformaciones monotónicas de los datos, heterocedasticidad condicional de los errores y colas pesadas.

Se parte de la serie de tiempo $y_{t}$ generada por una transformación monotónica como:

$y t=h(P t)$ 
$P t=P(P t-1, \ldots, P t-k)+u t, t=1, \ldots n$,

Donde $\left\{y_{t}\right\}, t=0, . . n$ es un conjunto de observaciones, $h\left(P_{t}\right)$ es una transformación monotónica desconocida de $\left\{P_{t}\right\}, P\left(P_{t-1}, \ldots, P_{t-k}\right)$ es un regresión desconocida de interés, $k$ es un entero positivo y $\left\{U_{t}\right\}$ es una secuencia de errores con mediana condicional igual a cero.

El interés de la prueba está enfocado en comprobar la hipótesis nula:

Ho: Pt=Pt-1+ut, $\quad t \geq 1$

En otras palabras, se trata de probar la hipótesis nula de caminata aleatoria versus la alternativa de un proceso explosivo lineal o no lineal bajo los supuestos 1 y 2 .

Supuesto 1. $\left\{\operatorname{signo}\left(u_{t}\right)\right\}$ es una secuencia de diferencias martingalas con respecto a una secuencia creciente de $\sigma$-anillos $\left\{F_{t}\right\}$ tal que $E\left[\operatorname{sign}\left(u_{t}\right) \mid\right.$ $\left.F_{t-1}\right]=0, t=1, \ldots, n$. .

Supuesto 2. $P\left[u_{t} \mid F_{t-1}\right]=0$,

De esta forma, el estadístico de signo es definido por So y Shin (2001):

$S_{n}(\rho)=\sum_{t=1}^{n} \operatorname{signo}\left(y_{t}-y_{t-1}\right)$

$\operatorname{signo}\left(y_{t-1}-\hat{m}_{t-1}\right) \geq n-2 B_{n}(\alpha)$

Si es ecuación se cumple, entonces se rechaza la hipótesis nula de caminata aleatoria expuesta en [3]. Signo $(\cdot)$ es el signo de $u_{t^{\prime}}$ y está definido como $\operatorname{Signo}\left(u_{t}\right)=1$ si $u_{t}>0 ;$ y $\operatorname{Signo}(0)=0$ y $\operatorname{Signo}\left(u_{t}\right)=-1$ si $u_{t}<0 ; \hat{m}_{t-1}$ es la mediana de $\left\{y_{t}\right\}_{t=0}^{n} ; B_{n}(\alpha)$ denota el $\alpha$-ésimo cuantil de la distribución binomial con parámetros $(n, 1 / 2) ; p=1$ y se trata de un proceso autoregresivo de orden 1 . Las pruebas de este teorema y de algunas de sus generalizaciones, así como las condiciones teóricas que deben ser satisfechas por las series en estudio para su correcta aplicación, son descritas en forma extensiva por So y Shin (2001).

Teniendo en cuenta las críticas de Evans (1991), se debe plantear la prueba de tal forma que pueda detectar burbujas que colapsan periódicamente. Para eso, la prueba de signo con ajuste recursivo de mediana es aplicada a submuestras que avanzan progresivamente, conocidas en la literatura como ventanas móviles. El tamaño de tales ventanas $(I)$, de mil datos, se define siguiendo el trabajo de Uribe (2013), que realiza simulaciones para determinar la potencia y la significancia empíricas de la prueba ante diferentes longitudes de las ventanas.

\section{Índice de burbujas}

Se puede definir el índice de burbujas como:

$$
\begin{aligned}
& B I_{t}=\left\{\begin{array}{cc}
S_{n t}-c v & \text { si } S_{n t}>c v \\
0 & \text { otro caso }
\end{array}\right. \\
& \forall t=0,1,2, \ldots, N-l
\end{aligned}
$$

Donde $B I_{t}$ corresponde al índice de burbujas para el periodo $t, N$ es el tamaño de la muestra y I es el tamaño de la ventana móvil (en este caso es igual a mil). El número $\mathrm{cv}:=\left[n-2 B_{n}(\alpha)\right]$ es el $\alpha$-iésimo percentil de la distribución binomial con parámetros $(I, 1 / 2)$. $S_{n t}$ es el estadístico definido en la ecuación [4] en el periodo $t$, con $p=1$. Entonces, se puede fechar el comienzo de la burbuja para el intervalo como:

$t_{B}^{i}=\left\{t_{j} \mid B I_{t}>0\right\}$

De igual manera, la fecha de colapso está dada por:

$t_{E}^{i}=\left\{t_{k} \mid B I_{t}=0, t_{k}>t_{j}\right\}$

Donde $t_{B}^{i}$ es el periodo $t_{j}$ que marca el comienzo de la burbuja, $t_{B}^{i}$ es el periodo $t_{k}$ que marca el final de la burbuja, $t_{k}$ y $t_{j}$ son números naturales tales que $t_{j}, t_{k} \in t$ e $i$ es un número natural que se usa para identificar la burbuja. Este procedimiento sirve para fechar múltiples burbujas en la serie, si existe evidencia de alguna.

\section{Análisis por componentes principales (PCA) y modelos de factores dinámicos (DFM)}

Para el análisis de las burbujas financieras y la construcción de los índices de mercado se hace uso de los modelos por factores. Siguiendo a Stock y 
Watson (2002a, 2002b, 2005) y Bai y Ng (2008), se construyen los modelos dinámicos por factores (DFM) para cada uno de los índices.

Así, se parte de $Y_{t^{\prime}}$ que es un vector de variables observables de $M \times 1$ para $t=1,2, \ldots T$ periodos, que recoge la dinámica de los diferentes mercados de acciones en el mundo. De esta manera, $Y_{t}$ contiene información de los diferentes índices y toma la mayor cantidad de información disponible sobre cada mercado. Se supone que existe información económica que está contenida en $Y_{t}$ y que es relevante en la explicación de la dinámica de las series de tiempo. Ese conjunto de información puede resumirse en un vector de factores no observables, $F_{t}$.

Si se parte de que $F_{t}$ se refiere a un proceso dinámico, es decir, sigue un proceso autorregresivo, se debe estimar el factor o los factores, haciendo uso de modelos que permitan captar este tipo de procesos. De acuerdo con el trabajo de Stock y Watson (2002a, 2005), estos factores se estiman siguiendo el modelo de factores dinámicos (DFM).

Según Stock y Watson (2002a), una serie de tiempo se puede expresar como una distribución de los rezagos de un conjunto más pequeño de factores comunes no observados, además de un error idiosincrático. Así, un factor se puede expresar como:

$X_{i t}=\lambda_{i}(L) f_{t}+u_{i t}$

Donde $X_{i t}$ representa el conjunto de series $i$ a los que se les desea reducir la dimensionalidad, $\lambda_{i}(\mathrm{~L})=\left[1-\lambda_{i 1} \mathrm{~L}-\ldots-\lambda_{i s^{2}}{ }^{s}\right]$ es el vector de cargas dinámicas de orden $S, f_{t}$ es el vector de factores inobservados y $u_{i t}$ corresponde a perturbaciones idiosincráticas. La dimensión de $f_{t}$ corresponde a la cantidad $q$ de factores dinámicos.

El modelo de factores dinámicos (DFM) se puede expresar como un modelo estático con $r$ factores:

$X_{t}=\Lambda F_{t}+e_{t}$

Donde $F_{t}$ tiene una mayor dimensión que $f_{t^{\prime}}$ dado que este incluye los factores dinámicos y sus $s$ rezagos. De esta forma, la cantidad de factores estáticos se puede expresar como $r=q(s+1)$. La elección de la cantidad de factores estáticos y dinámicos se realiza a partir de criterios de información presentados en Bai y $\mathrm{Ng}(2002,2008)$.

La estimación de los factores dinámicos se realiza a partir de análisis de componentes principales, lo cual permite reducir la cantidad de series, manteniendo una cierta cantidad de la variación de los datos originales. A partir de la matriz $X_{t^{\prime}}$ se construye la matriz $X_{c^{\prime}}$ que corresponde a la matriz original con los datos centrados con respecto a la media temporal. Partiendo de esta matriz, se calcula la covarianza muestral así:

$S=\frac{1}{n-1} X_{c}^{\prime} X_{C}$

De acuerdo con la matriz de covarianza $S$, se calculan los valores y vectores propios $\lambda_{j}$ y $\alpha_{j^{\prime}}$ respectivamente, de los cuales se puede calcular el $j$-esimo componente principal como:

$z_{j}=\alpha_{j}^{\prime}(x-\bar{x}) \quad \forall j=1, \ldots, n$

Donde $z_{j}$, el componente principal, no es más que una combinación lineal de las variables originales. Entonces, la matriz de componentes principales, $Z$ es la que corresponde a los scores de los componentes principales:

$Z=X_{c} A$

Dado que la matriz $Z$ todavía tiene la misma dimensión que la matriz $X_{c}$ original, se debe disminuir la cantidad de vectores propios de la matriz $A$, de tal forma que la matriz $Z$ calculada en [13] tenga una menor dimensión. Para esto se eligen los $r$ componentes principales con mayores valores propios asociados, es decir, que recoge la mayor cantidad de la varianza original. La selección de la cantidad de $r$ se realiza, como se mencionó, de acuerdo con criterios de información (Bai y Ng, 2008). De esta forma se puede escribir así:

$Z_{r}=X_{c} A_{r}$

Donde $A_{r}$ contiene los primeros $r$ valores propios y $Z_{r}$ es la matriz de scores de los componentes principales asociados con estos valores propios. 


\section{DATOS}

Para la construcción de las burbujas, se utilizaron cuarenta y seis (46) series de mercados financieros de todo el mundo. Para que las medidas fueran coherentes entre sí, se hizo uso del índice de MorganStanley ( $\mathrm{MSCl}$, por sus siglas en inglés) para cada uno de estos países, con frecuencia diaria, desde enero de 1995 hasta diciembre de 2013. Esto con el fin de analizar un total de 18 años disponibles. Los países analizados fueron: Estados Unidos, Japón, China, Alemania, Inglaterra, Brasil, India, Australia, Francia, Corea, Rusia, Italia, Singapur, Canadá, España, Suiza, Hong Kong, Indonesia, Malasia, México, Holanda, Sudáfrica, Suecia, Tailandia, Turquía, Austria, Bélgica, Chile, Egipto, Finlandia, Nueva Zelanda, Noruega, Polonia, Portugal, Taiwán, Argentina, Colombia, República Checa, Dinamarca, Hungría, Irlanda, Israel, Marruecos, Sri Lanka, Singapur, Pakistán y Perú. También se analizaron la Unión Motetaria Europea y el Índice Asiático ${ }^{1}$ (sin incluir Japón).

Tener índices de gran parte del mundo, 44 países y 2 regiones, permite analizar no solo individualmente sino por región los mercados financieros. Los índices se dividen en cinco grupos con base la región en la que se encuentran (tabla 1).

\section{RESULTADOS}

La mayoría de los mercados alrededor del mundo, desde inicios del siglo, han presentado un comportamiento homogéneo que puede dar cuenta de cierto grado de sincronización en sus ciclos financieros y en la aparición de burbujas. Al respecto, estudios como los de Gómez-González et al. (2014) han mostrado una reciente sincronía en los mercados financieros de Chile, Colombia y Perú, en especial para el caso de principios de siglo (burbuja punto-com) y en los años correspondientes a la crisis sub-prime en Estados Unidos.

1 Dentro de este índice se incluyen países como China, India, Indonesia, Corea, Malasia, Filipinas, Taiwán, Tailandia, Singapur y Hong Kong.
Esto se aúna al efecto que han tenido diversos choques financieros desde los Estados Unidos hacia el resto de las economías, en especial hacia Latinoamérica (Canova, 2005), para ayudar a configurar trayectorias similares de los precios en los mercados financieros globales. Al respecto, el trabajo de Uribe y Mosquera (2015) muestra que existe una alta sincronía, en los últimos años, en los ciclos financieros no solo en el ámbito regional, sino también en el mundial. Lo anterior llevaría a intuir que en materia de burbujas financieras, este fenómeno no debería ser diferente.

Como se puede observar en la gráfica 1, el mercado en general presentó un crecimiento

Tabla 1

\begin{tabular}{|c|c|c|c|c|}
\hline \multicolumn{5}{|c|}{ Índices por región. } \\
\hline \multicolumn{5}{|c|}{ Región } \\
\hline Norteamérica & Asia & Europa & Sudamérica & $\begin{array}{l}\text { Otros } \\
\text { paises }\end{array}$ \\
\hline $\begin{array}{l}\text { Estados } \\
\text { Unidos }\end{array}$ & Japón & Alemania & Brasil & India \\
\hline Canadá & China & Inglaterra & Chile & Australia \\
\hline México & Corea & Francia & Argentina & Rusia \\
\hline & Singapur & Italia & Colombia & Sudáfrica \\
\hline & $\begin{array}{l}\text { Hong } \\
\text { Kong }\end{array}$ & $\begin{array}{l}\text { España } \\
\text { Suiza }\end{array}$ & Perú & Turkía \\
\hline & Indonesia & Holanda & & Egipto \\
\hline & Malasia & Suecia & & $\begin{array}{l}\text { Nueva } \\
\text { Zelanda }\end{array}$ \\
\hline & Tailandia & Austria & & Israel \\
\hline & Taiwán & Bélgica & & Marruecos \\
\hline & Sri Lanka & Finlandia & & \\
\hline & Singapur & Noruega & & \\
\hline & & Polonia & & \\
\hline & & Portugal & & \\
\hline & & $\begin{array}{c}\text { República } \\
\text { Checa }\end{array}$ & & \\
\hline & & Dinarmarca & & \\
\hline & & Hungría & & \\
\hline & & Irlanda & & \\
\hline \multicolumn{5}{|c|}{ Índices regionales } \\
\hline La Unión & Monetaria & & $\begin{array}{r}\text { Índice Asiá } \\
\text { (sin incluir Ja }\end{array}$ & $\begin{array}{l}\text { tico } \\
\text { apón) }\end{array}$ \\
\hline
\end{tabular}

Fuente: elaboración de los autores. 
relativamente alto en el periodo comprendido entre 2001 y 2008, es decir, en el periodo posterior a la burbuja punto-com y hasta la crisis financiera sub-prime en Estados Unidos. En años anteriores, la relación entre los índices presentados parece no ser tan uniforme entre los países. Al respecto se puede argumentar que el grado de apertura y de globalización para estas épocas fue mucho menor.

Estados Unidos, Japón e Inglaterra presentan crecimientos y caídas consistentes con los periodos de crisis mundiales, con la diferencia de que Japón parece haber tardado más tiempo en recuperarse de la crisis financiera mundial, a pesar de los estímulos monetarios que han sido ejercidos por el respectivo banco central.

Los casos de Rusia y Colombia son un poco diferentes a los del resto de países. Antes del 2000 Colombia no tenía un mercado financiero definido y contaba con tres bolsas regionales diferentes. Solo en el 2001 se unieron estas y

Índices MSCI de países representativos por región

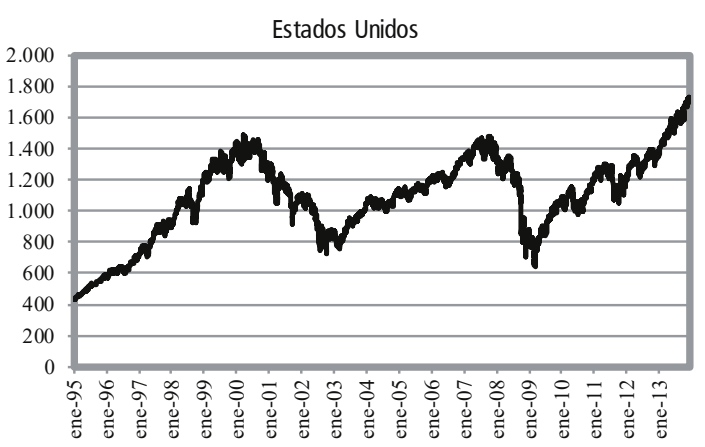

Japón

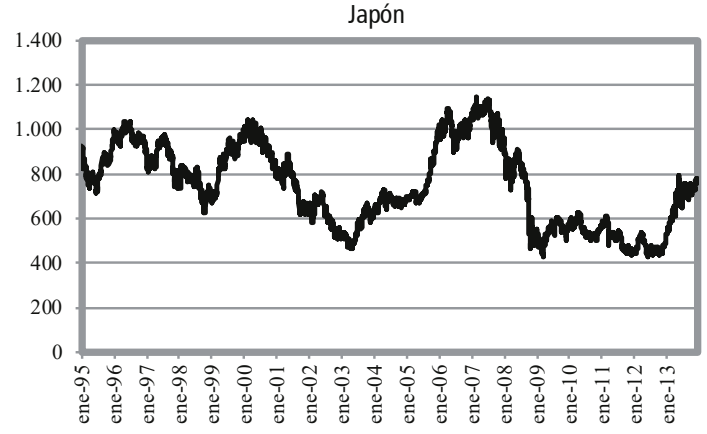

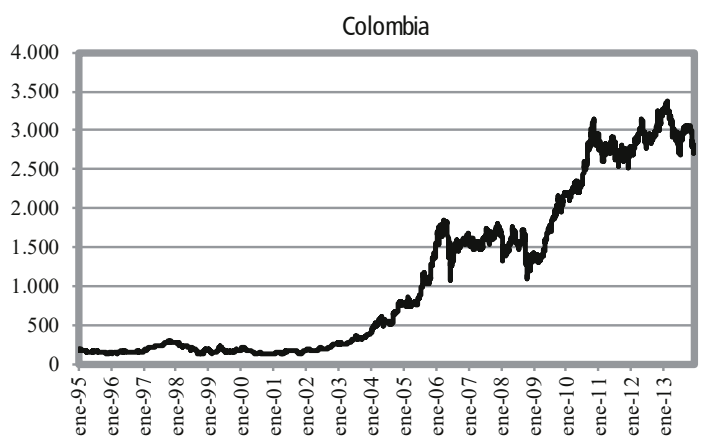

UK

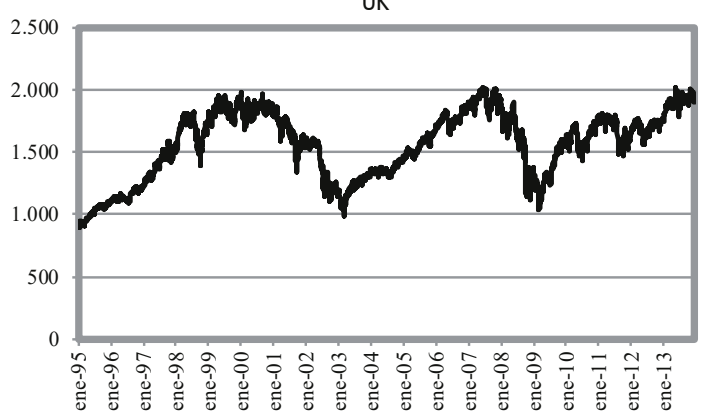

Rusia

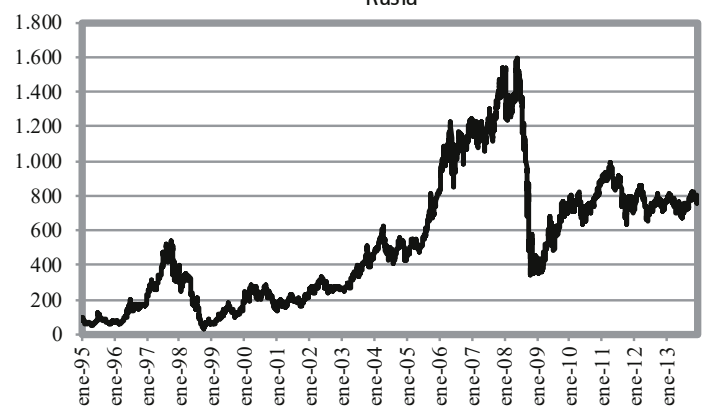

Fuente: Thompson Reuters Datastream. 
formaron una sola bolsa de valores, la Bolsa de Valores de Colombia (BVC); como se puede ver en la gráfica 1, desde ese entonces el mercado ha tenido un crecimiento exponencial. No obstante, este mercado sigue presentando problemas como concentración, baja liquidez del mercado y altas dependencias asintóticas (Martínez, 2008; Uribe y Fernández, 2014b; Uribe, Mosquera y Restrepo, 2013; Uribe, 2007). El efecto de las crisis financieras en Colombia no parece haber sido particularmente pronunciado ni persistente, ya que tal mercado además presentó un crecimiento acelerado en épocas posteriores. Rusia, por el contrario, presentó un gran crecimiento en las épocas previas a la crisis y, asimismo, registró una caída importante en el índice con posterioridad.

\section{Burbujas financieras mundiales}

Para contrastar la hipótesis de partida de este trabajo: la existencia de burbujas financieras simultáneas en diferentes regiones del mundo, se estimó un índice de burbujas que permitiera comparar a estos países y regiones. A cada índice de mercado de los países analizados en este trabajo se le aplicó el test de signo de So y Shin (2001) de manera recursiva, y posteriormente se calculó el índice de burbujas presentado por Uribe (2013), con un ancho de ventana de 1000 datos y un porcentaje de confianza del $95 \%$.

A partir de la gráfica 2 se puede concluir que existen periodos de burbujas comunes entre los países, en especial para el periodo de la burbuja sub-prime. Colombia, Rusia y Estados Unidos albergan periodos de crecimiento explosivo mayores a la de los demás países. Esto es especialmente cierto para Rusia, que presenta un proceso de marcado crecimiento explosivo desde el 2002, mucho antes de que iniciara un proceso análogo en Colombia, en el 2004.

La gráfica 3 contrasta cada una de las burbujas que se presentan en el mercado, en el tiempo analizado para los principales mercados regionales y para Colombia. Lo primero que se puede notar es que los resultados son coherentes con las teorías propuestas por Caballero, Farhi y Gourinchas $(2008,2009)$ sobre las crisis financieras. Los autores en mención argumentan que posterior a las crisis financieras en los países desarrollados (burbuja punto-com y crisis de Rusia), los flujos de capital migraron hacia economías con retornos más altos a la inversión. Así, estas economías sufrieron un crecimiento hasta el punto donde se agotaron las oportunidades de mercado. Dada la falta de desarrollo del mercado en estas regiones, estos capitales migraron de nuevo a las economías desarrolladas en busca de instrumentos financieros con mayor liquidez.

Gran parte de los argumentos sobre la sincronización de las burbujas en el periodo 2005-2008 radican en los excesos de liquidez, producto de las bajas tasas de interés en los países desarrollados, a inicios del siglo. Este exceso de liquidez se transformó en un flujo de inversiones en el escenario mundial que llenó de liquidez a los mercados mundiales. Una vez que llegó la crisis financiera en el 2007, los pánicos y la falta de confianza en el mercado llevaron a una disminución de los flujos y a una eventual contracción de todos los mercados.

Posterior a la crisis financiera, existe un periodo de corrección del mercado, en septiembre de 2008, consecuente con la bancarrota de Lehman Brothers. Un factor que puede generar preocupación sobre el futuro del mercado (gráfico 5) son las burbujas que se han ido formando en épocas posteriores a 2009. Estas, aunque se han mantenido en niveles ligeramente menores que las predecesoras, pueden dar señales sobre la necesidad de realizar políticas contractivas en el mercado para controlar su crecimiento y asimismo evitar una recesión de mayor volumen en el futuro.

Dado que no existen índices para cada mercado o estos presentan algunos limitantes - como el hecho de que no incluyen toda la información disponible o no se conoce bien como están midiendo el desempeño del mercado-, se debe construir un índice que tenga la capacidad de recopilar la 


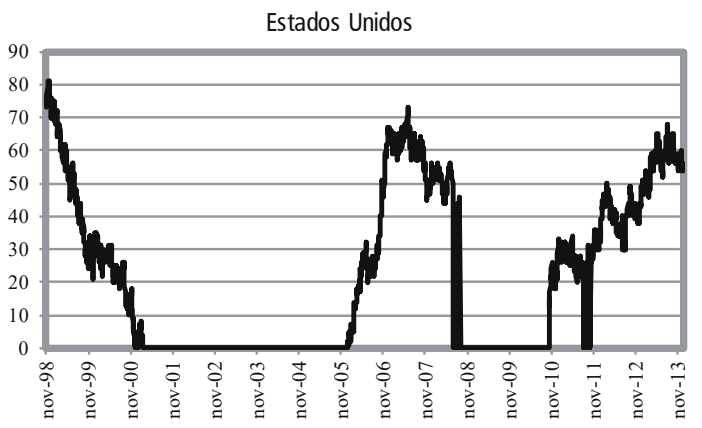

Japón

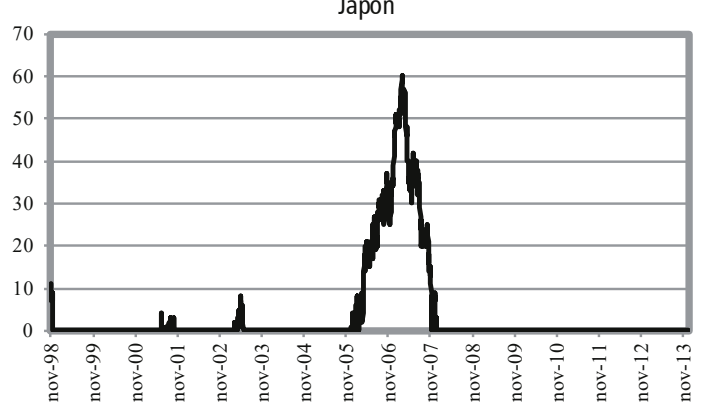

Colombia

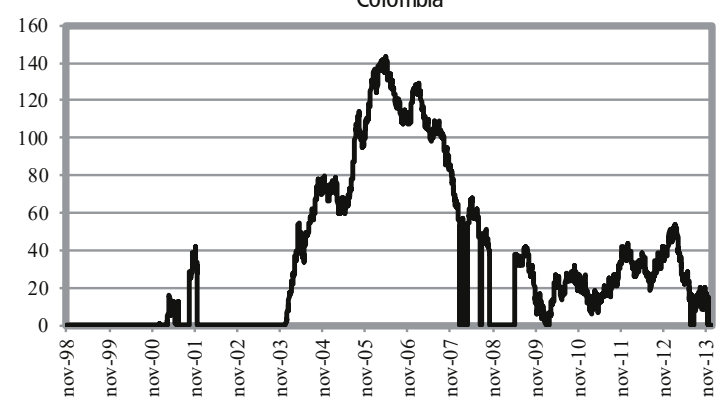

UK

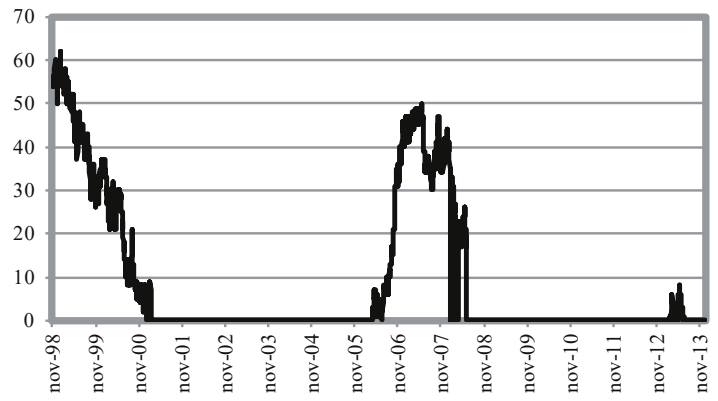

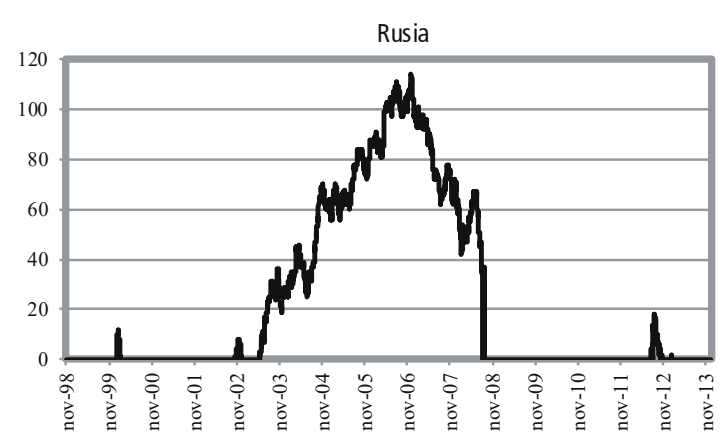

Fuente: elaboración de los autores.

información de todos los mercados. A continuación se presentan los índices construidos por factores, a partir de la metodología propuesta por Bai y $\mathrm{Ng}(2002,2008)$ y Stock y Watson (2002a, 2002b), por factores. Se espera así lograr una representación general del mercado de interés.

\section{Burbujas financieras en Norteamérica}

Para Norteamérica se construyó un índice que tiene en cuenta el comportamiento de los mercados estadounidense, canadiense y mexicano. Se espera que refleje la dinámica de las fluctuaciones del mercado de mayor tamaño y que, por lo tanto, provea mayores niveles de volatilidad, en este caso, el estadounidense.

Para Norteamérica existe una tendencia conjunta de las burbujas en el mercado. De las gráficas 5 y 6 se puede ver que el índice captura las burbujas financieras de los tres países. Este mercado, al estar tan compenetrado económicamente, tiene una alta relación, y de ahí deriva que el efecto de las burbujas sea tan uniforme. La burbuja financiera más marcada es la correspondiente 
Burbujas financieras del mundo

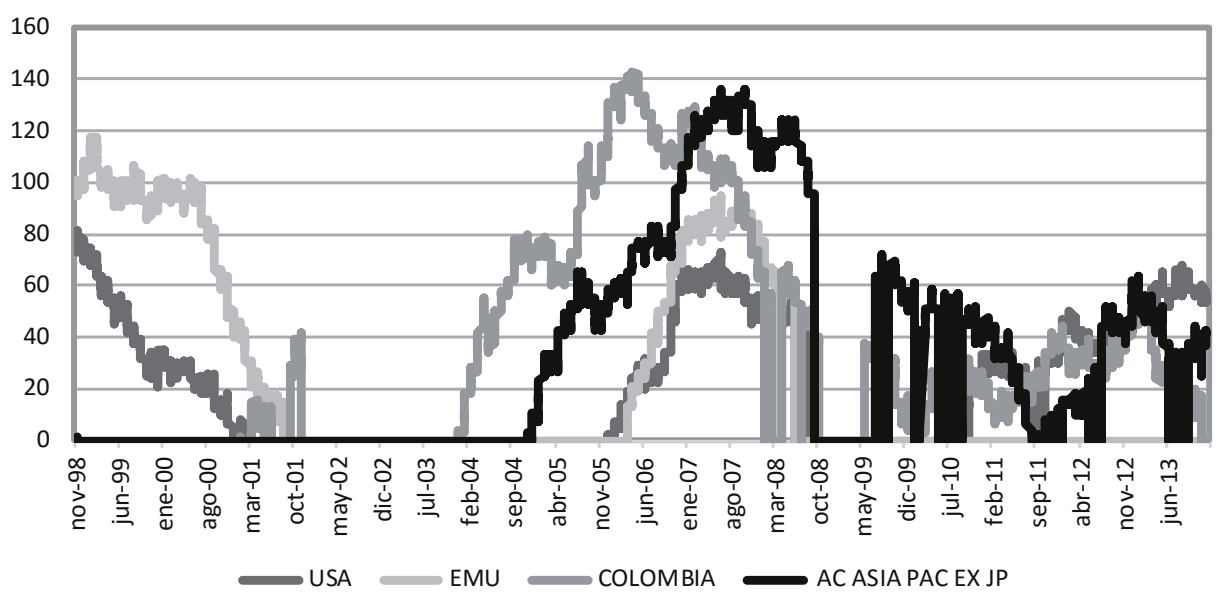

Nota: USA corresponde a Estados Unidos; EMU, a la Unión Europea; ASIA, a los países de la zona pacífica de Asia, sin incluir Japón. Fuente: elaboración de los autores.

Burbujas financieras, índices del mundo
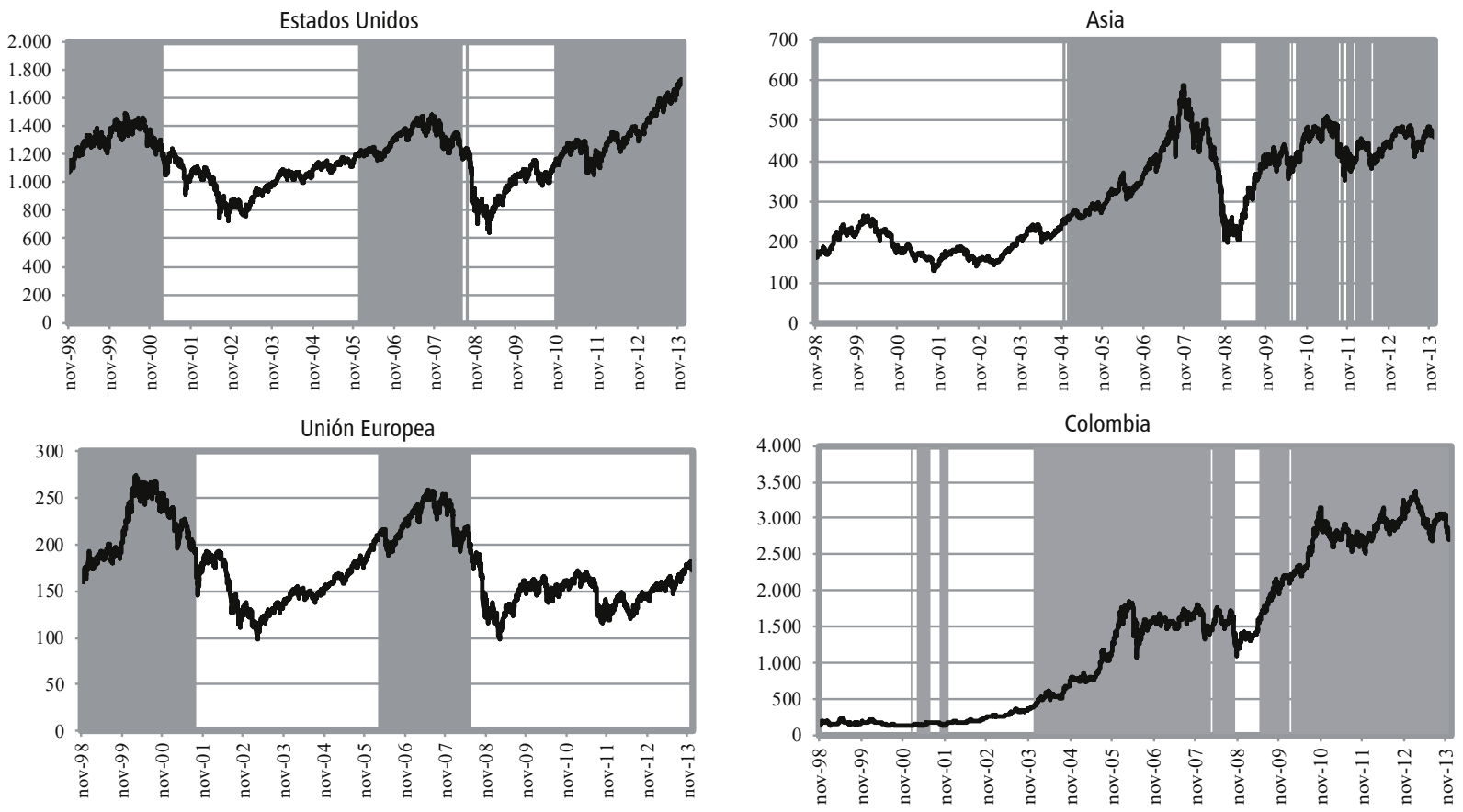

Fuente: elaboración de los autores. 
a la crisis sub-prime. Hay una nueva burbuja que está surgiendo desde mediados de 2009, especialmente en el mercado estadounidense.

Gráfica 5.

Burbujas en países de Norteamérica

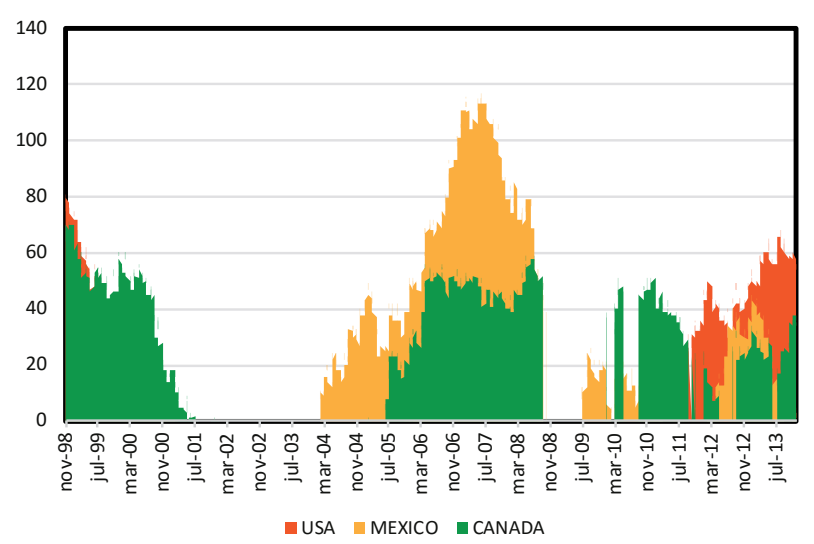

Fuente: elaboración de los autores.

\section{Grafica 6.}

Índice de burbujas en Norteamérica

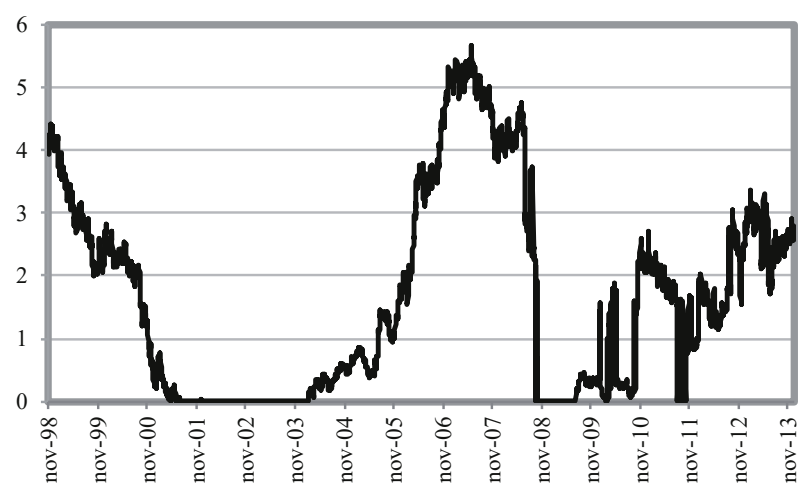

Fuente: elaboración de los autores.

\section{Burbujas financieras en Europa}

Para Europa, los índices que se tuvieron en cuenta fueron: Alemania, Reino Unido, Francia, Italia, España, Suiza, Holanda, Suecia, Austria, Bélgica, Finlandia, Noruega, Polonia, Portugal, República
Checa, Dinamarca, Hungría e Irlanda. El índice permite captar el efecto general, y no las dinámicas idiosincráticas asociadas con cada mercado.

Gráfica 7.

Burbujas en países de Europa

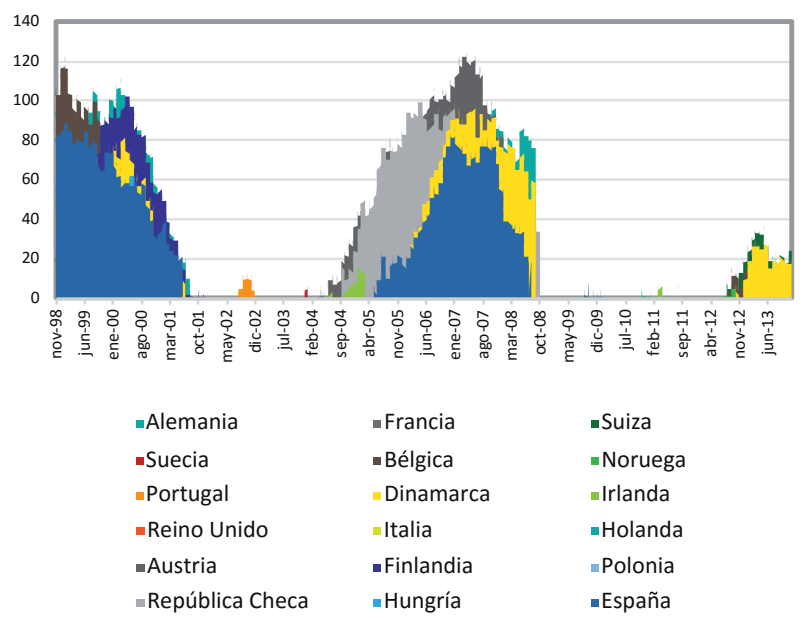

Fuente: elaboración de los autores.

Según la gráfica 7 , se puede ver que los mercados analizados son más homogéneos de lo que podría pensarse en primera instancia. Se puede conjeturar que la integración propia de la Unión Europea ha llevado a que los vínculos entre los países de la región se acrecienten y, por tanto, a que los choques se propaguen de manera uniforme. Lo anterior implica que los posibles beneficios de diversificación financiera, que permitirían teóricamente el acceso a diferentes mercados, con diferentes fundamentales asociados, se hayan visto reducidos en pro de una mayor integración financiera. Consistente con lo anterior, posterior a la crisis de 2007-2009 no se ha formado una nueva burbuja significativa. Esto puede derivarse del efecto persistente que ha tenido la crisis de la deuda soberana de algunos países de la Unión Europea, como España, Grecia, Portugal e Irlanda. La gráfica 8 deja entrever la mayor unión financiera de los mercados y la relación de las burbujas de finales de los noventa y la crisis de 2007. 
Gráfica 8 .

Índice de burbujas en Europa

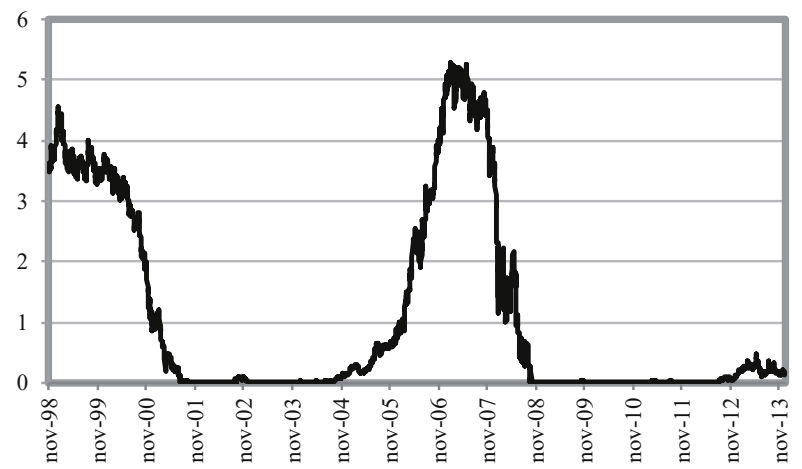

Fuente: elaboración de los autores.

\section{Burbujas financieras en Suramérica}

La construcción del índice por componentes principales para Suramérica considera los índices financieros de los países con los mercados más grandes y líquidos. Los índices tenidos en cuenta son: Argentina, Brasil, Chile, Colombia y Perú. Al ser países en desarrollo y con mercados más concentrados, se esperaría que existan altos niveles de volatilidad en el mercado y, además, burbujas marcadas. De acuerdo con los resultados obtenidos por Uribe y Fernández (2014a), se evidencia que durante el periodo que comprende los últimos años de la década de los noventa y el año 2007 existe una relación entre los mercados países sudamericanos, analizados en este trabajo, provenientes de los flujos de capital de los países desarrollados.

Los resultados obtenidos, presentados en las gráficas 9 y 10, confirman las hipótesis anteriores sobre los resultados esperados, en términos de sincronización de las burbujas financieras. Para finales de los años noventa y principios de siglo, existe una sincronización de las burbujas financieras, donde a medida que pasan crisis como la de Brasil, surgen nuevas crisis como la de Argentina. Colombia tiene una burbuja considerablemente marcada que inicia en el periodo posterior al 2002 (ver gráfica 1), en el cual tiene un crecimiento acelerado, al igual que la época posterior a la caída de septiembre de 2008.
Gráfica 9.

Burbujas en países de Suramérica

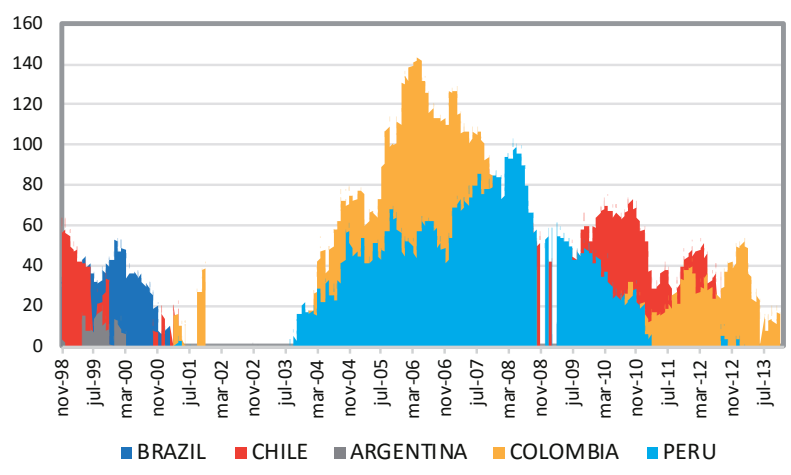

Fuente: elaboración de los autores.

El periodo posterior al 2008 también ha sido un periodo de burbuja para los demás países latinos, a pesar de no ser homogénea su magnitud. En términos generales, en esta época se puede observar una caída sostenida del periodo de burbuja.

Gráfica 10.

Índice de burbujas en Suramérica

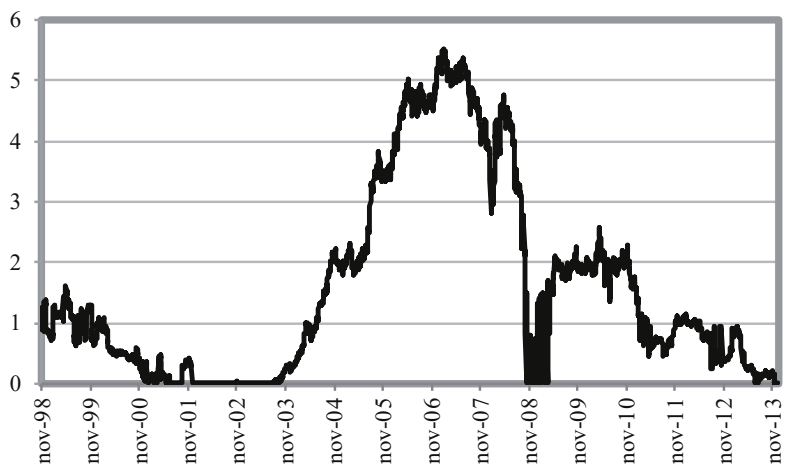

Fuente: elaboración de los autores.

\section{Burbujas financieras en Asia}

Más que cualquier otra región estudiada, Asia es considerablemente heterogénea y sus economías se han caracterizado por tener un conjunto de burbujas diferentes desde años atrás; la crisis de los 'tigres asiáticos' es una de las más grandes. Los países asiáticos utilizados para este análisis son: 
Japón, China, Corea, Hong Kong, Indonesia, Malasia, Tailandia, Taiwán, Sri Lanka y Singapur.

Gráfica 11 .

Burbujas en países de Asia

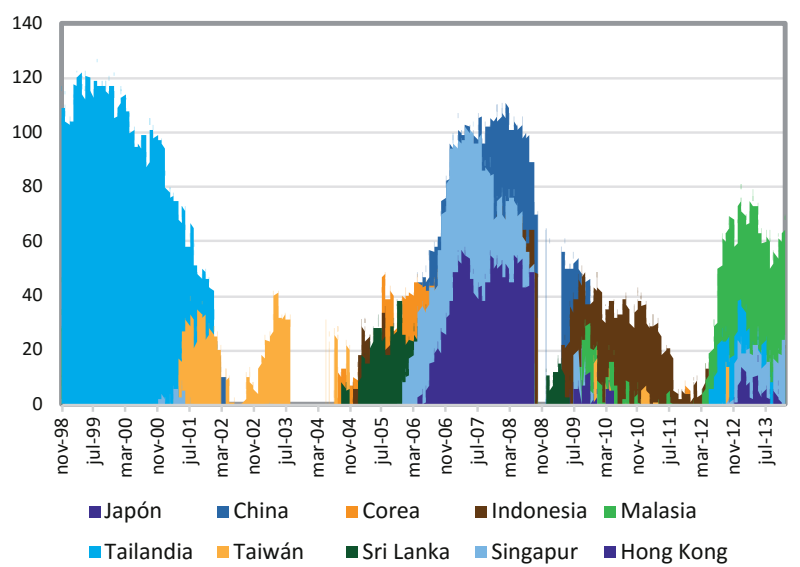

Fuente: elaboración de los autores.

La heterogeneidad de las burbujas en Asia, como se puede ver en las gráficas 11 y 12, lleva a que el índice de burbujas para esta región no presente un nivel de burbujas significativamente distinto al de las demás regiones en la muestra. Así, las burbujas en cada país no son capturadas en el índice general, pues se trata procesos explosivos con carácter idiosincrático.

Gráfica 12.

Índice de burbujas en Asia

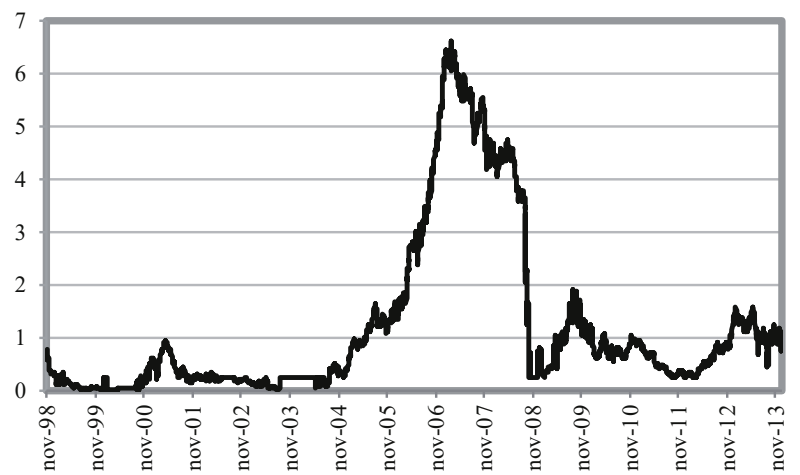

Fuente: elaboración de los autores.

\section{Burbujas financieras en el resto del mundo}

El resto de países (gráficas 13 y 14) corresponden a los países de India, Australia, Rusia, Sudáfrica, Turquía, Egipto, Nueva Zelanda, Israel, Marruecos y Pakistán. Dentro de los países estudiados destaca la burbuja de Marruecos, que se desarrolla en el periodo 20012004. Si esta burbuja se compara con el resto de los países durante la época, este resulta anormal. El crecimiento de este país está explicado por las reformas estructurales realizadas en el inicio de 2000 por el estado marroquí, para fomentar el crecimiento de la economía y su alta producción agrícola; y su caída, probablemente, se deba a la disputa entre este país y España por la soberanía de la isla Perejil.

Gráfica 13.

Burbujas en países del resto del mundo

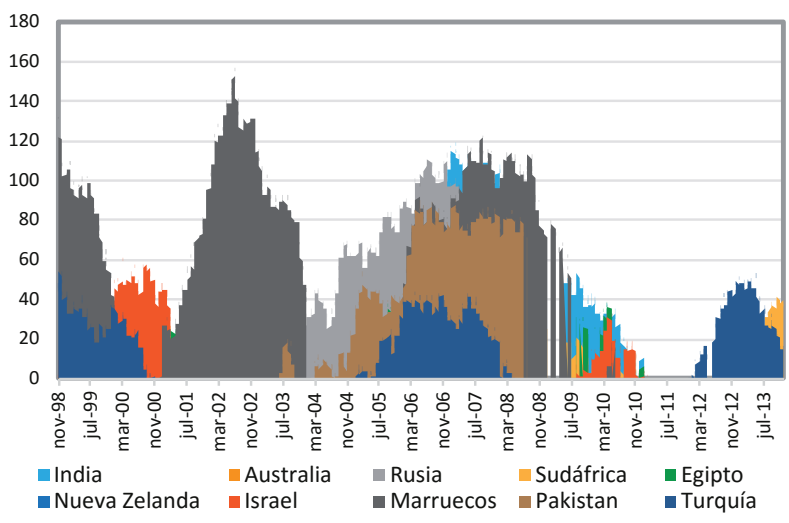

Fuente: elaboración de los autores.

Gráfica 14

Índice de países del resto del mundo

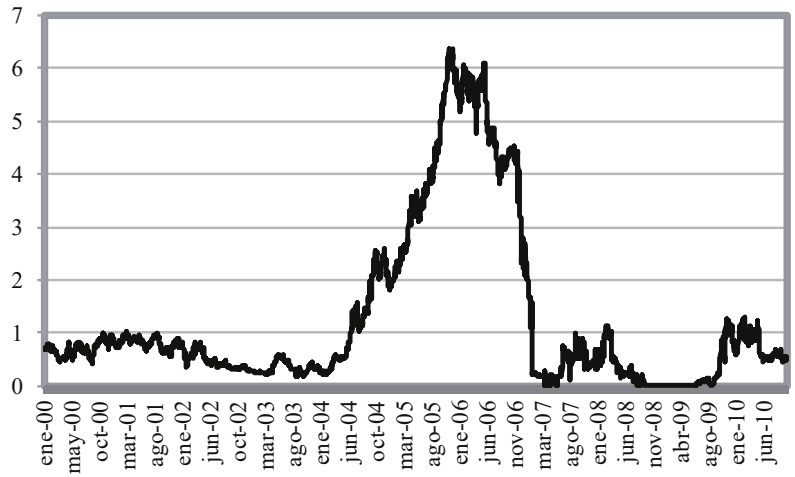

Fuente: elaboración de los autores. 


\section{Índice mundial de burbujas financieras}

A partir de los índices construidos previamente, se estima un índice mundial de burbujas financieras, para lo cual se usa una vez más la técnica de componentes principales. Este índice puede ser tomado como una medida de estabilidad económica global.

A partir de la gráfica 15 se puede concluir que han existido episodios conjuntos de inestabilidad económica y de burbujas financieras en el ámbito mundial. Estos episodios han estado marcados por una fuerte inestabilidad económica global; en este escenario, la crisis de 2007-2009 ha sido una de las más fuertes que se han experimentado. El índice permite determinar, además, la existencia de un periodo de sincronización de las burbujas mundiales, es decir, de periodos de crecimiento explosivo generalizado alrededor de los mercados de acciones globales.

Gráfica 15.

Índice de países del resto del mundo

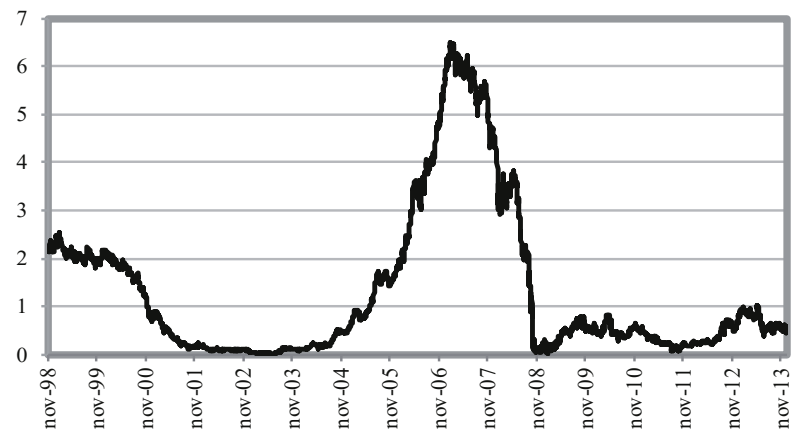

Fuente: elaboración de los autores.

Se debe tener en cuenta que episodios de "burbujas mundiales" son preocupantes. Primero, para los inversionistas y administradores de capital, pues teóricamente las posibilidades de diversificación se vuelven escasas y el riesgo de altas pérdidas es considerable. Los modelos de valoración llevan a la elección de activos con altos retornos y bajo riesgo, pero la inmensa mayoría de tales modelos no incluye alguna medida de burbujas o del grado de sobrevaloración de la cesta de activos financieros. Segundo, para quienes diseñan y ejecutan la política económica, pues en muchos casos los bancos centrales descartan totalmente el uso de algún modelo de medición de burbujas, para apoyar su toma de decisiones, y esto puede llevar a crecimientos excesivos de los precios financieros, que pueden desencadenar en crisis de proporciones ingentes.

\section{CONCLUSIONES}

Uno de los principales aportes de este estudio es la identificación de burbujas mundiales y la construcción de índices de burbujas para cada región y para el mundo. Los resultados aquí obtenidos son consecuentes con trabajos y desarrollos teóricos y empíricos de Caballero, Farhi y Gourinchas, (2008), Caballero et al. (2009) y Uribe y Fernández (2014a), quienes encuentran que existen sincronización entre las crisis financieras de los países desarrollados y los países en desarrollo, productores de commodities (materias primas). Además de estos trabajos, los resultados obtenidos son coherentes con los estudios de sincronización del ciclo financiero propuestos por Uribe y Mosquera (2015) y por Canova et al. (2005).

Las burbujas financieras halladas para cada uno de los mercados permiten dilucidar las características de cada economía y qué tan expuestas se encuentran ante choques externos. El efecto spillover de las crisis financieras provenientes de países desarrollados hacía países no desarrollados, como es el caso de Estados Unidos y los países latinoamericanos, pone en tela de juicio las conveniencias del libre flujo de inversión de capitales externos de corto plazo hacia las economías emergentes. La inversión externa es, entonces, conveniente en la medida que puede generar desarrollo de los mercados financieros y permite a las empresas captar ingresos, mas puede ser perjudicial dado el carácter temporal de los fondos invertidos, en términos de la generación de burbujas.

Los índices construidos marcan periodos en los que se hace necesario un seguimiento más cercano, por parte de las autoridades monetarias locales, de los mercados financieros de acciones. Ante un índice de burbujas general alto, se prenden 
las alarmas y se señala la necesidad de una mayor coordinación entre los bancos centrales mundiales, con miras a atenuar los efectos nocivos de estos fenómenos sobre las economías reales.

Para trabajos posteriores, se puede analizar más a fondo las razones de los comportamientos idiosincráticos de cada uno de los países aquí analizados y los determinantes de los periodos de burbujas financieras. La metodología posibilita la construcción no solo de medidas de burbuja en activos de diferente tipo, sino también de índices de burbuja sectoriales que permitan esclarecer qué sector está generando la burbuja financiera.

Finalmente, como posibles expansiones del análisis, se podría pensar en el efecto de la inversión directa de los países en el aumento de este índice y los mecanismos de transmisión sobre las regiones. Determinar estos mecanismos llevará a un análisis enriquecido sobre las políticas más eficientes en la prevención de crisis futuras.

\section{REFERENCIAS}

1. Akerlof, G. A. y Shiller, R. J. (2009). Animal spirits: how human psychology drives the economy, and why it matters for global capitalism. Nueva Jersey: Princeton University Press.

2. Allen, F. y Gale, D. (2000). Bubbles and crises. Economic Journal, 110(460), 236-255. Doi: $10.1111 / 1468-0297.00499$

3. Anderson, K. y Brooks, C. (2014). Speculative bubbles and the cross-sectional variation in stock returns. International Review of Financial Analysis, 35, 20-31. Doi: 10.1016/j.irfa.2014.07.004

4. Anderson, K., Brooks, C. y Katsaris, A. (2010). Speculative bubbles in the S\&P 500: Was the tech bubble confined to the tech sector? Journal of Empirical Finance, 17(3), 345-361. Doi: doi.org/10.1016/j. jempfin.2009.12.004

5. Bai, J. y Ng, S. (2002). Determining the number of factors in approximate factor models. Econometrica. Doi: $10.1111 / 1468-0262.00273$

6. Bai, J. y Ng, S. (2008). Forecasting economic time series using targeted predictors. Journal of Econometrics, 146(2), 304-317. Doi: 10.1016/j.jeconom.2008.08.010

7. Brunnermeier, M. K. y Oehmke, M. (2013). Bubbles, financial Crises, and systemic risk. Recuperado de https://www0.gsb.columbia.edu/faculty/moehmke/papers/BrunnermeierOehmkeHandbookSystemicRisk. pdf

8. Caballero, R. J., Farhi, E. y Gourinchas, P.-O. (2008). An equilibrium model of "global imbalances" and low interest rates. The American Economic Review, 98(1), 358-393. Doi: 10.1257/aer.98.1.358

9. Caballero, R. J., Farhi, E. y Gourinchas, P.-O. (2009). Financial crash, commodity prices, and global imbalances. Brookings Papers on Economic Activity. Doi: http://doi.org/10.1353/eca.0.0013

10. Campbell, J. Y. y Shiller, R. J. (1988a). Stock prices, earnings, and expected dividends. Journal of Finance, 43(3), 661-676. Doi: 10.2307/2328190

11. Campbell, J. Y. y Shiller, R. J. (1988b). The dividend-price ratio and expectations of future dividends and discount factors. Review of Financial Studies, 1 (3), 1-34. Doi: 10.1093/rfs/1.3.195

12. Canova, F. (2005). The transmission of US shocks to Latin America. Journal of Applied Econometrics, 20(2), 229-251. Doi: doi.org/10.1002/jae.837

13. Corsi, F. y Sornette, D. (2014). Follow the money: The monetary roots of bubbles and crashes. International Review of Financial Analysis, 32, 47-59. Doi: 10.1016/j.irfa.2014.01.007 
14. Diba, B. T. y Grossman, H. I. (1987). On the Inception of Rational Bubbles. The Quarterly Journal of Economics, 102(3), 697-700. Doi: 10.2307/1884225

15. Diba, B. T. y Grossman, H. L. (1988a). Explosive rational bubbles in stock prices? American Economic Review, 78(3), 520-530. Doi: 10.2307/1809149

16. Diba, B. T. y Grossman, H. L. (1988b). The Theory of Rational Bubbles in Stock Prices. The Economic Journal, 98(392), 746-754. Doi: 10.2307/2233912

17. Dickey, D. A. y Fuller, W. A. (1979). Distribution of the estimators for autoregressive time series with a unit root. Journal of the American Statistical Association, 74(366), 427-431. Doi: 10.2307/2286348

18. Evans, G. W. (1991). Pitfalls in testing for explosive bubbles in asset prices. American Economic Review, 81(4), 922-930. Doi: 10.2307/2006651

19. Farmer, R. E. a. (2012). Confidence, crashes and animal spirits. Economic Journal, 122 (559), 155-172. Doi: $10.1111 /$ j.1468-0297.2011.02474.x

20. Froot, K. A. y Obstfeld, M. (1991). Intrinsic bubbles: the case of stock prices. American Economic Review, 81(5), 1189-1214. Doi: 10.1257/aer.89.5.1372

21. Gómez-González, J. E., Ojeda-Joya, J. N., Zárate, H. M. y Tenjo-Galarza, F. (2014). Testing for causality between credit and real business cycles in the frequency domain: an illustration. Applied Economics Letters, 21(10), 697-701. Doi: 10.1080/13504851.2014.884689

22. Gürkaynak, R. S. (2008). Econometric tests of asset price bubbles: Taking stock. Journal of Economic Surveys, 22(1), 166-186. Doi: 10.1111/j.1467-6419.2007.00530.x

23. Hall, S., Psaradakis, Z. y Sola, M. (1999). Detecting periodically collapsing bubbles: a markovswitching unit root test. Journal of Applied Econometrics, 14(2), 143-154. Doi: 10.1002/ (SICI) 1099-1255(199903/04) 14:2<143::AID-JAE500>3.0.CO;2-X

24. Hall, S. y Sola, M. (1993). Testing for collapsing bubbles: test, an endogenous Switching ADF (Discussion Paper 15-93, London Business School).

25. Hamilton, J. (1989). A new approach to the economic analysis of nonstationary time series and the business cycle. Econometrica, 57(2), 357-384. Doi:10.2307/1912559

26. Homm, U. y Breitung, J. (2012). Testing for speculative bubbles in stock markets: A comparison of alternative methods. Journal of Financial Econometrics, 10(1), 198-231. Doi: 10.1093/jjfinec/nbr009

27. Keynes, J. M. (1936). The general theory of emplyment, interest and money. Londres: MacMillan.

28. Leiss, M., Nax, H. H. y Sornette, D. (2015). Super-exponential growth expectations and the global financial crisis. Journal of Economic Dynamics and Control, 55, 1-13. Doi: 10.1016/j.jedc.2015.03.005

29. LeRoy, S. F. y Porter, R. D. (1981). The present-value relation: tests based on implied variance bounds. Econometrica, 49(3), 555-574. Doi: 10.2307/1911512

30. Malevergn, Y. y Sornette, D. (2006). Extreme financial risks: from dependence to risk management. extreme financial risks: from dependence to risk management. Doi: http://doi.org/10.1007/b138841

31. Martinez, C. (2008). The effects of financial intermediation on Colombian economic growth. Revista Ensayos Sobre Política Económica, 26(57), 250-280.

32. Phillips, P. C. B., Shi, S. y Yu, J. (2014). Specification sensitivity in right-tailed unit root testing for explosive behaviour. Oxford Bulletin of Economics and Statistics, 76(3), 315-333. Doi: 10.1111/obes.12026 
33. Phillips, P. C. B., Shi, S.-P. y Wu, Y. (2014). Testing for Multiple Bubbles: Historical Episodes of Exuberance and Collapse in the SEPP 500. Recuperado de http://cowles.econ.yale.edu/P/cd/d19a/d1914.pdf

34. Phillips, P. C. B., Wu, Y. y Yu, J. (2011). Explosive Behavior In The 1990S Nasdaq: When did exuberance escalate asset values? International Economic Review, 52 (1), 201-226. Doi: 10.1111/j.1468-2354.2010.00625.x

35. Phillips, P. C. B. y Yu, J. (2011). Dating the timeline of financial bubbles during the subprime crisis. Quantitative Economics, 2(3), 455-491. Doi: 10.3982/QE82

36. Samuelson, P. A. (1958). An exact consumption-loan model of interest with or without the social contrivance of Money. Journal of Political Economy, 66(6), 467-482.

37. Shell, K. (1971). Notes on the Economics of Infinity. Journal of Political Economy, 79(5), 1002. Doi: $10.1086 / 259811$

38. Shi, S. (2007). Moving window unit root test: locating real estate price bubbles in Seoul apartment market. Recuperadode http://ink.library.smu.edu.sg/etd_coll/28/

39. Shiller, R. J. (1981). Do stock prices move too much to be justified by subsequent movements in dividends? American Economic Review, 71(3), 421-436.

40. Shiller, R. J. (2005). Irrational exuberance (2. ${ }^{\text {a }}$ ed.). Broadway Books.

41. So, B. S. y Shin, D. W. (2001). An invariant sign test for random walks based on recursive median adjustment. Journal of Econometrics, 102 (2), 197-229. Doi: 10.1016/S0304-4076(01)00053-7

42. Sornette, D. (2009). Dragon-kings, black swans, and the prediction of crises. International Journal of Terraspace Science and Engineering, 2(1), 1-18. Doi: 10.2139/ssrn.1470006

43. Sornette, D., Woodard, R. y Zhou, W. X. (2009). The 2006-2008 oil bubble: Evidence of speculation, and prediction. Physica A: Statistical Mechanics and its Applications, 388(8), 1571-1576. Doi: 10.1016/j. physa.2009.01.011

44. Stock, J. H. y Watson, M. W. (2002a). Forecasting using principal components from a large number of predictors. Journal of the American Statistical Association, 97(460), 1167-1179. Doi: 10.1198/016214502388618960

45. Stock, J. H. y Watson, M. W. (2002b). Macroeconomic forecasting using diffusion indexes. Journal of Business Economics and Statistics, 20(2), 147-162.

46. Stock, J. H. y Watson, M. W. (2005). Understanding changes in international bussiness cycle dynamics. Journal of the European Economic Association, 3(5), 968-1006.

47. Tirole, J. (1982). On the possibility of speculation under rational expectations. Econometrica, 50(5), 1163 1182. Doi: $10.2307 / 1911868$

48. Tirole, J. (1985). Asset bubbles and overlapping generations. Econometrica, 53(6), 1499-1528. Doi: $10.2307 / 1913232$

49. Uribe, J. M. (2007). Caracterización del mercado accionario colombiano, 2001-2006: un análisis comparativo. Recuperado de http://www.banrep.gov.co/sites/default/files/publicaciones/pdfs/borra456.pdf

50. Uribe, J. M. (2013). Testing for multiple bubbles with daily data. Recuperadode http://www.banrep.gov.co/ sites/default/files/publicaciones/archivos/sem_301.pdf

51. Uribe, J. M. y Fernández, J. (2014a). Burbujas financieras y comportamiento reciente de los mercados de acciones en América Latina. Lecturas de Economía, (81), 57-90.

52. Uribe, J. M. y Fernández, J. (2014b). Riesgo sistémico en el mercado de acciones colombiano: alternativas de diversificación bajo eventos extremos. Cuadernos de Economía, 33(63), 613-634. 
53. Uribe, J. M. y Mosquera, S. (2015). A comparative analysis of stock market cycles. Macroeconomics and Finance in Emerging Market Economies. (en prensa)

54. Uribe, J. M., Mosquera, S. y Restrepo, N. X. (2013). Mercado de Acciones Colombiano. Determinantes macroeconómicos y papel de las AFP. Sociedad y Economía, 24, 207-230.

55. Uribe, J. M. y Ulloa, I. (2014). Burbujas financieras : dos alternativas de identificación aplicadas a Colombia. Sociedad y Economía, (27), 47-72.

56. van Norden, S. (1996). Regime switching as a test for exchange rate bubbles. Journal of Applied Econometrics, 11 (3), 219-251. Doi: 10.1002/(SICI) 1099-1255(199605) 11:3<219::AID-JAE394>3.0.CO;2-S

57. van Norden, S. y Vigfusson, R. (1998). Avoiding the Pitfalls: Can Regime-Switching Tests Reliably Detect Bubbles? Studies in Nonlinear Dynamics $\mathcal{G}$ Econometrics. Doi: 10.2202/1558-3708.1038

58. West, K. D. (1987). A Specification Test for Speculative Bubbles. Quarterly Journal of Economics, 102 (3), 553-580. Doi: $10.2307 / 1884217$

59. West, K. D. (1988). Dividend Innovations and Stock Price Volatility. Econometrica, 56(1), 37-61. Doi: $10.2307 / 1911841$

60. Wu, Y. (1997). Rational bubbles in the stock market: accounting for the U.S. Stock-price volatility. Economic Inquiry, 35(2), 309-319. Doi: 10.1111/j.1465-7295.1997.tb01912.x

61. Yuhn, K.-H., Kim, S. B. y Nam, J. H. (2015). Bubbles and the Weibull distribution: was there an explosive bubble in US stock prices before the global economic crisis? Applied Economics, 47(3), 255-271. Doi: $10.1080 / 00036846.2014 .969824$

62. Zhou, W. X. y Sornette, D. (2003). Renormalization group analysis of the 2000-2002 anti-bubble in the US S\&P500 index: Explanation of the hierarchy of five crashes and prediction. Physica A: Statistical Mechanics and its Applications, 330(3-4), 584-604. Doi: 10.1016/j.physa.2003.09.022

63. Zhou, W. X. y Sornette, D. (2005). Testing the stability of the 2000 US stock market "antibubble". Physica A: Statistical Mechanics and its Applications, 348, 428-452. Doi: 10.1016/j.physa.2004.09.032 